Citation: V. Ferro Allodola (2020) Fake news e forme di dialogo online e offline: diventare resilienti attraverso la Media Literacy. Media Education 11(1): 67-75. doi: 10.36253/me-9096

Received: February 2020

Accepted: May 2020

Published: July 2020

Copyright: () 2020 V. Ferro Allodola. This is an open access, peer-reviewed article published by Firenze University Press (http://www.fupress.com/me) and distributed under the terms of the Creative Commons Attribution License, which permits unrestricted use, distribution, and reproduction in any medium, provided the original author and source are credited.

Data Availability Statement: All relevant data are within the paper and its Supporting Information files.

Competing Interests: The Author(s) declare(s) no conflict of interest.

\section{Fake news e forme di dialogo online e offline: diventare resilienti attraverso la Media Literacy}

\author{
Fake news and forms of online and offline dialogue: becoming \\ resilient through Media Literacy
}

\author{
Valerio Ferro Allodola \\ Ateneo Telematico "eCampus" \\ E-mail: valerio.ferroallodola@uniecampus.it
}

\begin{abstract}
Moving from the definition of fake news and its application to the first studies in the field, especially in the Italian context, this paper aims at reflecting on violent forms of online and offline dialogue today, also referring to the recent spread of Covid-19, an extremely complex situation in which we have been able to "touch closely" the importance of having access to true and reliable information to adopt adequate health behaviors. At the same time, this contribution intends to examine how to promote users' awareness of responsible use of the internet and their resilience through Media Literacy, by referring to national and international training initiatives, studies and research on the topic.
\end{abstract}

Keywords: fake news, online and offline violence, resiliency, media literacy.

\begin{abstract}
L'articolo, partendo dalla definizione di fake news e dall'applicazione del concetto ai primi studi nel settore, specie nel contesto italiano, si propone di riflettere sulle forme violente di dialogo online e offline oggi, anche in riferimento alla recente diffusione del Covid-19, situazione di estrema complessità nella quale abbiamo potuto "toccare da vicino" l'importanza di poter avere accesso a informazioni attendibili per adottare comportamenti di salute adeguati. Allo stesso tempo, il presente contributo intende approfondire come responsabilizzare gli utenti ad un uso consapevole di internet e diventare resilienti mediante la Media Literacy, facendo riferimento a iniziative formative, studi e ricerche nazionali e internazionali sul tema.
\end{abstract}

Parole chiave: fake news, violenza online e offline, resilienza, media literacy.

\title{
INTRODUZIONE
}

La rapidità della comunicazione, oggigiorno, gioca a sfavore della capacità di riflessione e di approfondimento, producendo una seria difficoltà a distinguere - spesso - il vero dal falso, tanto che post-truth (post-verità) è stata la parola dell'anno del 2016 secondo gli studiosi degli Oxford Dictionnaries (Gili et al., 2018; Trinchero, 2018). 
Con il costrutto inglese fake news si indicano "quelle informazioni costruite che imitano i contenuti dei media nella forma ma non nel processo organizzativo o nell'intento. Tali notizie false, a loro volta, mancano di norme e processi editoriali dei media per garantire l'accuratezza e la credibilità dell'informazione. Le notizie false si sovrappongono ad altri disturbi informativi, come la misinformazione (informazioni false o fuorvianti) e la disinformazione (informazioni false che sono volutamente diffuse per ingannare le persone)" (Lazer et al., 2018, p. 1094).

Il contesto politico è stato il primo ad essere affrontato e studiato (assieme alle vaccinazioni e all'alimentazione) (Ibidem), sia perché è enorme l'impatto delle decisioni politiche sulla vita delle persone, sia per capire se e quanto le fake news avessero contribuito a orientare il dibattito $o$, addirittura, influito più o meno direttamente sui risultati. Quello che ha spinto a indagare il fenomeno da un punto di vista teorico è stata l'ampiezza della sua diffusione come mai prima, complice un generale clima di sfiducia e di delegittimazione delle istituzioni, dei media tradizionali e la volontà delle persone di disintermediare la ricerca di informazioni e di crearsi un'opinione in maniera autonoma (Cosentino, 2017).

Vero, falso e verosimile s'intrecciano senza sosta, contribuendo a far vacillare meccanismi tradizionali di formazione dell'opinione pubblica, di percezione e costruzione della realtà, facendo venire meno il concetto stesso di narrazione condivisa, producendo narrazioni tra loro contrastanti (Ferro Allodola, 2020).

Uno studio statunitense (Vosoughi et al., 2018), ad esempio, ha segnalato che una fake news su Twitter viene in genere ritwittata da molte più persone e molto più rapidamente delle informazioni vere, specialmente quando l'argomento è la politica. Weedon et al. (2017) hanno stimato che, in Facebook, le manipolazioni delle informazioni effettuate da attori malintenzionati rappresentino meno di un decimo dell'1\% dei contenuti civici condivisi sulla piattaforma, sebbene non siano stati pubblicati i dettagli di questa analisi.

È proprio sulla responsabilità di un certo tipo di giornalismo che si continua a riflettere (Sorrentino, 2018), anche da un punto di vista etico: de-contestualizzazione delle notizie, velocizzazione dei tempi nei contenuti da produrre, commercializzazione della professione sono fattori che hanno causato una crisi di credibilità senza precedenti, che ha generato anche l'opinione certamente estrema, ma piuttosto diffusa, che del giornalismo e dei giornalisti si possa tranquillamente fare a meno.

Ireton et al. (2018) hanno redatto una guida per l'UNESCO, dal titolo "Journalism, Fake News \& Disinformation". Rivolta ad esperti del settore, essa elenca i principi essenziali del buon giornalismo:
- accuratezza nel raccogliere le notizie;

- indipendenza da qualsiasi tipo di interesse;

- equità;

- confidenzialità per quanto riguarda le fonti;

- umanità;

- accuratezza nel riportare fatti;

- trasparenza.

Ciò che è stata definita un'infodemia ${ }^{1}$, conosciuta anche come "bulimia informativa" (Gori, 2018), ha incrementato la complessità anche nella gestione dell'attuale emergenza di Covid-19, pregiudicando l'opportunità di diffondere istruzioni chiare, precise ed ottenendo, conseguentemente, comportamenti omogenei da parte della popolazione. Rispetto alle emergenze globali e non solo sanitarie del passato, tutto questo rappresenta un fenomeno "di rottura": prima dell'avvento di internet e dei social network, infatti, la minore velocità di circolazione delle notizie e la carenza di mezzi di comunicazione consentivano una reazione più ordinata di fronte $\mathrm{a}$ fenomeni di portata globale (Grandi et al., 2020).

Il miglioramento dell'informazione può e deve certamente passare da un giornalismo più attento, che verifichi meglio le fonti e che non diffonda notizie false come se fossero vere, ma è soprattutto necessaria una formazione all'(auto)consapevolezza degli utenti stessi.

Quello che occorre è una svolta pedagogico-educativa nell'uso e nella gestione più consapevole dei dati e delle informazioni che oggi sono alla portata di tutti. Non si tratta di acquisire competenze tecniche o di imparare a usare i social network, ma di apprendere come valutare fatti e posizioni e verificarne veridicità $\mathrm{e}$ attendibilità. Strumenti tecnologici di debunking, ossia di smascheramento delle fake news, possono risultare utili: ad esempio vi sono strumenti di Google ${ }^{2}$ o di TinEye ${ }^{3}$ che permettono di verificare le immagini; Flip feed è il plug-in di Twitter che fa apparire tweet presi in modo casuale (mostrando così anche idee diverse); Escape your bubble è un plug-in di Facebook per leggere anche contenuti che normalmente non vedremmo in base all'algoritmo. Esistono anche motori di ricerca che non tracciano l'utente, come ad esempio, DuckDuck Go.

\footnotetext{
${ }^{1}$ Con questa espressione si intende la "Circolazione di una quantità eccessiva di informazioni, talvolta non vagliate con accuratezza, che rendono difficile orientarsi su un determinato argomento per la difficoltà di individuare fonti affidabili”. Enciclopedia Treccani online. http:// www.treccani.it/vocabolario/infodemia \%28Neologismi\%29/

${ }^{2}$ Google è un motore di ricerca il cui dominio è stato registrato il 15 settembre 1997 ed è il sito più visitato al mondo. Oltre a catalogare e indicizzare le risorse del World Wide Web, Google Search si occupa anche di foto, newsgroup, notizie, mappe (Google Maps), email (Gmail), shopping, traduzioni, video e programmi creati da Google.

${ }^{3}$ TinEye è un motore di ricerca in grado di trovare immagini simili ad una immagine preventivamente selezionata dall'hard disk o da internet, indicandone l'url nel campo predisposto. http://www.tineye.com/
} 
Inoltre, sia Google sia Facebook stanno lavorando sul fronte del fact checking, ossia sul controllo della veridicità dei fatti. Ma è di tutta evidenza che, se non si attua un mutamento culturale che incentivi chi intenda approfondire e acquisire coscienza critica, qualsiasi tipo di strumento è, se non inutile, quantomeno insufficiente. Sicuramente una certa critica al sistema di comunicazione e informazione può essere utile, ma occorre anche ammettere che ci troviamo in un ritardo culturale enorme e cominciare a porre in atto delle soluzioni, lavorando in primis sulla formazione e sulla Media Literacy (d'ora in poi M.L.) (Baacke, 1997; Buckingham, 2005; Cappello et al., 2018; Cappello, 2019; Felini, 2004; Hobbs, 2010; Livingstone, 2004; Ranieri et al., 2019; Silverstone, 2007). Sicuramente scuola e università (con molta più esperienza Oltralpe) si adoperano da tempo come attori di questo mutamento culturale.

Come già evidenziato da Cappello (2019), la M.L. in Italia ha una lunga tradizione. Gli attori principali provengono dalla società civile (accademici, associazioni, comunità ecclesiali, insegnanti, professionisti dei media, educatori), con un supporto crescente da parte delle istituzioni locali e nazionali. Mentre l'alfabetizzazione digitale è stabilmente entrata nel sistema scolastico attraverso fondi, progetti e risorse didattiche volte a migliorare le competenze digitali degli studenti, solo di recente ha sviluppato una visione più socioculturale delle tecnologie digitali, grazie anche al Piano nazionale per la scuola digitale pubblicato dal MIUR nel 2015. Per il futuro, è necessario un maggiore impegno per lo sviluppo delle seguenti tre importanti direzioni: condurre ricerche valutative sulla M.L., al fine di avere una conoscenza basata sull'evidenza del suo impatto sui contesti educativi, sia formali che non formali; migliorare la formazione e la produzione di risorse di qualità per insegnanti ed educatori; favorire un maggiore coordinamento e collegamento in rete tra le varie parti interessate e coinvolte nel settore (Ibidem).

A livello internazionale, ad esempio, la Finlandia ha inserito la M.L. e l'insegnamento al pensiero critico nel piano scolastico nazionale nel 2016 a partire dalla scuola Primaria; questo rappresenta un ottimo esempio di come un governo può agire se vuole combattere contro la diffusione di notizie false senza ricorrere a controverse leggi "anti fake news" (Mackintosh et al., 2018) Anche il governo britannico ha elaborato un programma educativo contro il caos informativo, inviando giornalisti nelle scuole per aiutare bambini e ragazzi a un consumo consapevole delle notizie (House of Commons Digital, Culture, Media and Sport Committee, UK, 2019).

Secondo i dati raccolti dall'European Policies Initiative, Open Society Institute (Lessenski, 2018), in uno stu- dio che ha misurato "la capacità di resilienza al fenomeno della 'post-verità', delle fake news e delle conseguenze annesse, cercando di offrire degli strumenti utili a trovare possibili soluzioni" (Lessenski, 2018, p. 2), l'Italia si classifica al ventunesimo posto. Prima tra tutti i 35 Paesi partecipanti allo studio è risultata la Finlandia (Lessenski, 2018, p. 3). L'educazione sembra essere la migliore soluzione a tutto tondo per promuovere la resilienza alle fake news e al fenomeno della post-verità: promuove il buon senso e responsabilizza. I risultati dell'Indice di M.L. indicano che un'istruzione di alta qualità e persone sempre più istruite costituiscono dei prerequisiti per affrontare gli effetti negativi delle fake news e del fenomeno della post-verità. Inoltre, sembra esistere una correlazione tra qualità dell'istruzione e libertà dei media quando vengono confrontati i due indicatori con l'Indice. I Paesi con una migliore istruzione tendono ad avere più libertà dei media e viceversa. Mentre l'Indice non può individuare causa ed effetto, l'osservazione stessa di una relazione è indicativa (Lessenski, 2018, pp. 10-14).

\section{VIOLENZA IN RETE: FORME DI DIALOGO ONLINE E OFFLINE}

Le fake news da sole non spiegano il continuo peggioramento del clima della comunicazione online e offline, specialmente dopo l'avvento e la diffusione del Covid-19 in Italia. Questo evento, infatti, in seguito alle misure restrittive adottate dal Governo - quarantena e distanziamento sociale, in primis, complice anche la comunicazione istituzionale inizialmente poco chiara ha prodotto nelle persone disorientamento, stress e forme di intolleranza sociale.

Spesso le fake news si sposano con messaggi pieni di odio e rancore, che diventano un "tono di voce" accettabile e permesso anche sui media mainstream come stampa, tv e radio e non solo sui social network. Ciò che prima veniva stigmatizzato e considerato inaccettabile, ora è all'ordine del giorno e si può trovare ovunque, in un programma di intrattenimento, così come nell'esternazione di qualche politico: l'Italia vanta infatti un triste primato in Europa sui livelli dell'odio in rete, come affermato in occasione del World Speech Day, la giornata mondiale della parola che si è tenuta per la prima volta in Italia a marzo 2018 a Firenze.

L'hate speech, il linguaggio che trasuda odio, è più diffuso di quello che si pensa. Risentimento, sdegno e intolleranza si dispiegano contro singole persone o gruppi specifici per i motivi più disparati: razziali, etnici, religiosi, di genere o orientamento sessuale (Nicodemo, 2017; Pasta, 2018). Sebbene il termine sia molto diffuso, non è 
definito univocamente (Article 19, 2015). La Corte Europea dei Diritti dell'Uomo, in una definizione adottata dal Comitato dei Ministri del Consiglio d'Europa, considera l'hate speach come: "tutte le forme di espressione che diffondono, incitano, promuovono o giustificano l'odio razziale, la xenofobia, l'antisemitismo o altre forme di odio basate sull'intolleranza, compresa l'intolleranza espressa dal nazionalismo aggressivo e dall'etnocentrismo, dalla discriminazione e dall'ostilità nei confronti di minoranze, migranti e persone di origine immigrata". YouTube, nelle sue linee guida della community, descrive l'hate speech come: "contenuti che promuovono violenza o odio contro individui o gruppi basati su determinati attributi quali: razza o origine etnica, religione, disabilità, genere, età, stato di veterano o orientamento sessuale/identità di genere". Il Comitato internazionale delle Nazioni Unite per l'eliminazione della discriminazione razziale concepisce l'hate speech come "una forma di discorso eterodiretto che rifiuta il nucleo dei principi dei diritti umani della dignità umana e dell'uguaglianza, mirata a degradare la posizione di individui e gruppi e il loro apprezzamento da parte della società". Infine, la Commissione per i Reclami in materia di Radiodiffusione in Sud Africa identifica l'hate speech come "tutto quel materiale che, giudicato nel contesto di sanzioni, promuove o amplifica la violenza basata su razza, origine nazionale o etnica, colore, religione, genere, orientamento sessuale, età, disabilità mentale o fisica", oppure "la propaganda per la guerra; l'istigazione alla violenza; la difesa dell'odio basato su razza, etnia, genere o religione e che costituisce un incitamento a causare danni" (Article 19, 2015, p. 12).

Il $51^{\circ}$ Rapporto sulla situazione sociale del Paese del Censis (2017) precisa che i gruppi più svantaggiati sono quelli che si sentono meno rappresentati e più inclini a posizioni populiste e a toni più accesi.

L'atteggiamento sull'immigrazione è una cartina di tornasole significativa e al contempo preoccupante. Il 59\% degli italiani la giudica negativamente, e più si appartiene a una classe sociale bassa, più la percentuale di negatività sale: arriva al $72 \%$ tra le casalinghe, al $71 \%$ tra i disoccupati, al 63\% tra gli operai (Censis, 2017).

A questo si aggiunge che tutti i soggetti politici italiani soffrono di gravi crisi di fiducia: il primato negativo va ai partiti politici, di cui l' $84 \%$ degli italiani non si fida, ma anche le istituzioni nazionali e locali non godono di miglior salute: il Governo ha il $78 \%$ di sfiducia, il Parlamento il 76\%, Regioni e Comuni il 70\% (Censis, 2017, p. 32).

Ancora: "il 60\% degli italiani si dichiara insoddisfatto di come funziona la democrazia nel nostro Paese; il $64 \%$ è convinto che la voce del cittadino semplicemente non conti nulla" (Ibidem).
Anche le campagne elettorali e referendarie nostrane non sono state esenti da fake news e da toni pesanti: durante la campagna referendaria l'opinione pubblica si è spaccata a metà tra i sostenitori della riforma e i suoi critici con toni spesso inutilmente aggressivi e virulenti, che hanno condizionato le interazioni nell'ambiente dei social e non solo (Nicodemo, 2017).

Questo clima eccessivamente rabbioso ha nuociuto alla qualità del dibattito pubblico, anche a causa dei tanti cosiddetti troll, ovvero "dei soggetti che interagiscono con gli altri tramite messaggi provocatori, irritanti, fuori tema o semplicemente senza senso e/o del tutto errati, con il solo obiettivo di disturbare la comunicazione e fomentare gli animi" (Jansen \& James, 2014, p. 386) che disturbano le conversazioni politiche e non solo.

Esistono quattro categorie di troll, ma tutti sono ugualmente dannosi (Ibidem):

- chi provoca comportamenti molesti;

- chi ama essere scandaloso e causare confusione;

- chi adora discutere;

- chi è troppo stupido per sapere di essere un troll.

$\mathrm{Ma}$ al di là del protagonismo di singoli troll, la nota dolente è che si assiste impotenti all'avvelenamento del dibattito politico pubblico che trascendono sino ad arrivare all'insulto, all'offesa, all'oltraggio vero e proprio.

La situazione generale sembra essere sconfortante, anche se una prima parziale, ma importante risposta, è stata data dal manifesto della comunicazione non ostile elaborato nel 2017. Un impegno di responsabilità condivisa per creare una Rete rispettosa e civile, che ci rappresenti e che ci faccia sentire in un luogo sicuro. Scritto e votato da una community di oltre 300 comunicatori, blogger e influencer, è una carta con 10 principi utili a ridefinire lo stile con cui stare in Rete ${ }^{4}$.

Naturalmente, un manifesto, per quanto ben scritto e condivisibile, non può bastare: purtroppo la consapevolezza contro fake news e odio online è diminuita invece che aumentare. Con il 2018 si è infatti ridotto il livello di allarme e di attenzione dei cittadini sull'hate speech. Secondo una ricerca dell'SWG di Trieste ${ }^{5}$, presentata nella seconda edizione di Parole Ostili a Trieste nel giugno 2018, a essere preoccupati per la deriva rancorosa che ha preso il linguaggio, in rete e non solo, sono il 53\% degli italiani, contro il 70\% del 2017. Cala anche l'allarme sulle fake news, ma in maniera più ridotta: il 59\% dei cittadini contro il $65 \%$ del 2017. Pare evidente una certa tendenza all'assuefazione, con un conseguente calo di attenzione di massa e di perdita di interesse.

\footnotetext{
${ }^{4}$ Cfr. http://paroleostili.com/manifesto/

${ }^{5}$ Cfr. https://www.ansa.it/canale_lifestyle/notizie/societa_diritti/2018/06/07/italia-assuefatta-no-allarme-hate-speech_ef741ffe-44164c2c-84d1-3f3f1287f59b.html
} 
Anche in questo caso, la chiave di volta per reagire all'antipolitica e al rancore è pedagogica e culturale. Le professionalità della comunicazione sono molto spesso inadeguate perché formate in maniera insufficiente a comprendere i meccanismi dell'impatto della comunicazione (Sensi, 2017). Formazione, definizione dei profili a cui aspirare, programmazione e riprogrammazione della comunicazione online e offline, creazione di community e partecipazione costruttiva: da queste quattro sfere di sviluppo passa la rigenerazione della comunicazione (Sensi, 2017).

Vi sono tentativi di autoregolamentazione, come quello proposto dall'Autorità per le Garanzie nelle Comunicazioni (AGCOM, 2018), con particolare attenzione al dibattito pubblico sui temi dell'immigrazione.

È necessario anche intervenire a livello di base, nei confronti di tutti gli utenti, potenziandone le competenze digitali - a partire dalla scuola primaria - per evitare che i meccanismi di odio si autoalimentino e che le persone diventino veicoli di rancore.

Consultando i rapporti dell'International Press Institute (2019), troviamo ancora la Finlandia tra i paesi meno impregnati dalla cattiva informazione, pur essendo un Paese esposto a fake news sugli stessi temi che vedono vittime il resto dei paesi europei, Italia compresa: immigrazione e campagne d'odio di stampo razzista.

In realtà, già nel 1968, il governo finlandese creò il "Council for Mass Media", un comitato di autoregolamentazione dei mezzi di comunicazione di massa, che aveva il potere di vincolare (non censurare, ma rettificare, si noti) una testata giornalistica a pubblicare una rettifica nei casi di violazione delle buone prassi professionali. Oggi, in Finlandia esistono dei funzionari del governo impegnati proprio in specifici corsi per combattere la disinformazione, dei giornalisti che, oltre a fornire indicazioni su come evitare le fake news, insegnano agli studenti, ai genitori e ai docenti, come leggere le notizie con una modalità critico/riflessiva (Cappello et al., 2018; Ranieri et al., 2018).

Addirittura, alcuni fat checkers digitali finlandesi hanno iniziato a collaborare con i sistemi scolastici, portando alla creazione di alcuni manuali d'uso (Neuvonen et al., 2018) molto importanti per la formazione al contrasto delle fake news e al diritto ad un sistema di informazione sano.

\section{DIVENTARE RESILIENTI ATTRAVERSO LA MEDIA LITERACY}

Gli individui tendono, generalmente, a non mettere in discussione la credibilità delle informazioni a meno che esse non violino i loro preconcetti o sono incentiva- ti a farlo. Le informazioni, altrimenti, vengono accettate in modo acritico, anche perché le persone tendono ad allineare le loro credenze con i valori della comunità di appartenenza.

La ricerca di Swire et al. (2017) dimostra, a riguardo, che le persone preferiscono le informazioni che confermano i loro atteggiamenti preesistenti (esposizione selettiva), considerare le informazioni coerenti con le loro convinzioni preesistenti come più persuasive di informazioni dissonanti (bias di conferma) e sono propense ad accettare le informazioni che le soddisfano (bias di desiderabilità) (Ibidem).

Precedenti credenze e ideologie potrebbero impedire il controllo dei fatti contenuti in una notizia falsa. Tuttavia, il controllo dei fatti potrebbe anche essere controproducente in determinate circostanze. Infatti, la ricerca sulla fluidità e il pregiudizio di familiarità nei dibattiti politici mostra che le persone tendono a ricordare le informazioni o la loro posizione rispetto ad esse, dimenticando il contesto in cui le hanno incontrate. Inoltre, gli individui hanno maggiori probabilità di accettare informazioni familiari come vere (Ibidem), con il conseguente rischio che la ripetizione di informazioni false in un contesto di verifica dei fatti, potrebbe aumentare la probabilità individuale di accettarle come vere.

Come già evidenziato altrove (Ferro Allodola, 2020), gli studiosi parlano di vere e proprie camere dell'eco o echo-chambers (Quattrociocchi et al., 2016), grazie a cui l'utente vede più facilmente contenuti simili ai propri. Si innesca, in tal modo, un effetto di risonanza che si autoalimenta e che viene utilizzato consciamente o inconsciamente per confermare la propria posizione. Oltre alle camere dell'eco vi è il concetto della bolla-filtro, la filter bubble (Nicodemo, 2017; Veltri, 2018) che di fatto isola l'utente, perché l'algoritmo dei social non lascia filtrare niente che non sia conforme ai gusti e alle convinzioni dell'utente stesso.

Come dimostrato da Lewandosky et al. (2017) le correzioni alle fake news sono efficaci solo quando sono soddisfatte almeno due condizioni: in primo luogo, esse non devono sfidare direttamente le visioni del mondo delle persone; ad esempio, è possibile incoraggiare l'autostima dei destinatari delle correzioni, utilizzando anche mezzi grafici. In secondo luogo, le correzioni devono spiegare al meglio perché la disinformazione è stata diffusa e devono fornire una spiegazione alternativa dell'evento messo in discussione.

A tal proposito, si rivela interessante il "Debunking Handbook" (http://sks.to/debunk), un libro per sfatare scientificamente le false credenze su alcuni argomenti di rilevante importanza, ad esempio lo scetticismo sul riscaldamento globale. 
Consideriamo, anche, che la diffusione di fake news sta facendo attecchire tra i giovani una cultura del sospetto e dell'incertezza: la metà degli studenti intervistati nella ricerca di Newman e coll. (2018) teme di non riuscire a riconoscere una fake news e oltre la metà degli insegnanti $(60,9 \%)$ ritiene che le fake news esercitino una influenza negativa sul benessere degli studenti, alimentando ansia e sfiducia in sé stessi. A crescere, è inoltre la sfiducia dei giovani verso il mondo dei news media: il $60 \%$ degli under 18 guarda alle notizie che circolano sui media di informazione con scetticismo e rassegnazione (Ibidem).

Sicuramente, la problematica delle fake news, della disinformazione e della disinformazione non può essere trattata in modo semplicistico. Questo articolo intende evidenziare la necessità di contestualizzare e connettere tali fenomeni con il più ampio contesto politico, tecnologico e sociale in cui essi hanno luogo.

Da uno studio recente di Buccolo et al. (2020) su percezioni e vissuti emotivi al tempo del Covid19 - con un campione composto da oltre 5.000 persone in tutta Italia - si evince, ad esempio, che il $37,2 \%$ utilizza siti web ufficiali delle Istituzioni per aggiornarsi sulla diffusione e le conseguenze del Covid-19 e il 37\% utilizza, invece, la TV. Solo l' $8,4 \%$ i social network, i quotidiani il $6,4 \%$, il $5 \%$ le riviste scientifiche e la restante percentuale la radio, internet e amici e conoscenti.

Questo dato, effettivamente, deve farci riflettere, poiché siti istituzionali e TV hanno ottenuto praticamente lo stesso punteggio. Interpretare questo dato, potrebbe voler significare che molte persone considerano la TV attendibile quanto i siti governativi, oppure che la TV rimane semplicemente il mezzo di comunicazione più diffuso e soprattutto più accessibile.

Dal database di Eurostat (2020) notiamo, però, che la percentuale di utenti abituali del web che fanno riferimento prioritariamente ai canali di comunicazione istituzionale è sensibilmente inferiore (19\%) a quella di altri paesi europei, come la Germania (58\%) o la Francia (48\%). Questo ulteriore dato ci spinge a pensare che l'infodemia possa essere particolarmente diffusa nel nostro Paese.

Se consideriamo che dall' 8 all' 11 marzo sono stati emanati tre DPCM e che esiste il cosiddetto "indice di leggibilità", possiamo pensare che i decreti siano accessibili a chi ha un livello di istruzione alto (laurea) e se consideriamo che l'Italia si classifica come uno dei Paesi con minor tasso di laureati (dati ISTAT 2016: n. 311.791; fanalino di coda tra i 28 Paesi UE, al penultimo posto) ${ }^{6}$

\footnotetext{
${ }^{6}$ Cfr. http://dati.istat.it/Index.aspx?DataSetCode=DCIS_LAUREATI\#; per quanto riguarda il confronto dell'Italia con gli altri Paesi dell'U.E.; https://www.ansa.it/europa/notizie/rubriche/altrenews/2020/04/22/euro-
}

e con più anziani (dati ISTAT aprile 2020: n. 7.058.755 75 anni e più), possiamo ben comprendere la limitata efficacia comunicativa di questi interventi istituzionali e quindi il fatto che molte persone preferiscano i programmi televisivi per informarsi. Pensiamo anche soltanto alla terminologia utilizzata negli ultimi mesi, anche in inglese: lockdown, assembramento, i nomi per le varie tipologie di mascherine da indossare, ecc. Non tutti sanno, ad esempio, che non si deve parlare di "Coronavirus" al singolare, perché questi sono una famiglia di virus a cui appartiene il Covid-19 (ISS, 2020). Questo, naturalmente, soltanto per fare degli esempi sulla complessità della comunicazione oggi.

Molto interessante, a riguardo, è il concetto di "rappresentazione sociale" (Farr et al., 1989), espressa nel caso del COVID-19 in diversi modi: inizialmente $\mathrm{i}$ media tradizionali e i social media parlavano di "virus di Wuhan" o "virus cinese diffusosi nel mercato di Wuhan", spesso attivando nella popolazione italiana diffidenza verso i cinesi residenti in Italia e verso i viaggi da e per il nostro Paese.

Successivamente all'esplosione del contagio in Italia, è mutata anche la terminologia con la quale la si definisce: "guerra", "soldati al fronte", "eroi" ecc., quasi come a volersi difendere dagli invasori cinesi. Cadalanu (2020), ad esempio, scrive: "Il governo cinese ha tenuto nascoste le dimensioni dell'epidemia per almeno un mese. Ma è inutile sottolineare che le responsabilità non si spalmano su un'intera nazione e tanto meno su un intero continente, basandosi magari su elementi come il colore della pelle. E invece il pregiudizio è diventato virale: su Twitter, per esempio, è un florilegio di giochi di parole razzisti, usando le parole Flu (vuol dire influenza) e Lung (polmone): da "Chop Fluey" a "Mao Tse Lung", da "Toflu" fino a "Kung Flu", espressione che viene attribuita a funzionari della Casa Bianca. Purtroppo, ci sono stati fenomeni di razzismo contro le comunità cinesi in Italia (Faloppa, 2020).

La rappresentazione di un fatto, di un evento, di una problematica assume rilevanza per l'individuo "perché definisce contemporaneamente cosa costituisce una realtà per il soggetto (il soggetto reagisce alla realtà non in quanto tale, ma in base a come se la rappresenta) e perché essa produce anticipazioni e aspettative che predeterminano quale sarà la relazione che il soggetto avrà con l'ambiente" (Ferro Allodola, 2013, p. 125).

$\mathrm{Da}$ un punto di vista educativo e formativo, contrastare le fake news, ma anche le notizie incomplete e obsolete, richiede non solo ricerca scientifica, ma una programmazione effettiva relativa all'inserimento

stat-italia-si-conferma-penultima-in-ue-per-laureati-_bf72a534-d540415b-9268-9d2b5a471679.html 


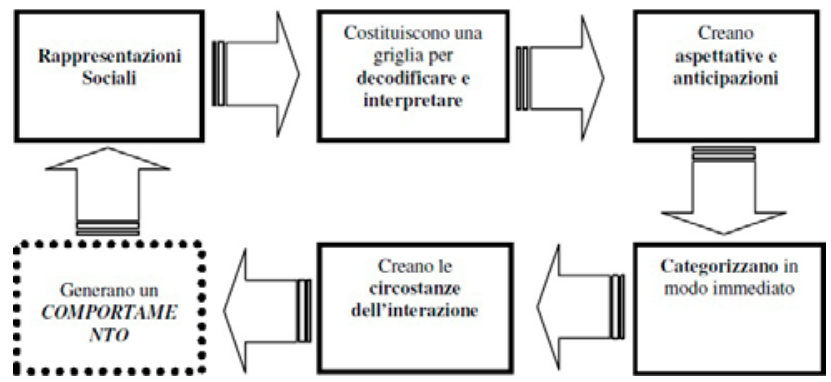

Figura 1. Schema tratto da Ferro Allodola V. (2013), p. 125.

di possibili moduli ad hoc circa la responsabilizzazione all'uso consapevole dei media, a partire dai curricola della Scuola Primaria e in tutti gli ordini e gradi di scuola. Certamente, non si deve trascurare la Terza Età, visto che questa rappresenta una fascia d'età abbastanza vulnerabile alle notizie false e con insufficienti competenze informatiche. Nel nostro Paese, ad esempio, oltre a una rosa di corsi formativi per anziani, è stato predisposto da Federanziani (2020) un'utile Vademecum dedicato, in particolare, a come salvaguardare sé stessi e gli altri in questo periodo di Covid-19.

Su un fronte più ampio e tecnologico, si iniziano ad intravedere le prime ricerche sulla possibilità di costruire delle infrastrutture per consentire agli utenti di Internet la gestione delle notizie false e di altri fenomeni mediatici online (Rehm, 2018).

Un recente studio (Stanford History Education Group, 2016) ha osservato che le responsabilità che prima erano dei redattori e dei bibliotecari, sono ora sulle spalle di chiunque usi uno schermo per essere informato sul mondo. Come per qualsiasi altro progresso nella tecnologia, la risposta non è togliere questi diritti-doveri ai comuni cittadini, ma formarli insegnando loro come impegnarsi nella ricerca di informazioni e nella valutazione delle notizie e delle informazioni (Ibidem). Ciò che si rende urgentemente necessario è poter trovare fonti credibili su tutti i fronti, da siti Web affidabili e ben strutturati per la diffusione delle notizie quotidiane, alle informazioni accademiche, agli studi di ricerca clinica.

A livello universitario, ad esempio, è responsabilità delle istituzioni accademiche, in particolare delle biblioteche, corroborare le competenze di M.L. per preparare adeguatamente gli intellettuali e i leader del futuro.

La M.L., oltre alla capacità di valutare la credibilità di una risorsa informativa, rafforza anche le capacità metacognitive sviluppando il pensiero critico e la capacità di ragionamento degli studenti. Il ruolo della biblioteca accademica è, dunque, quello di rafforzare sempre più la preparazione degli studenti, affinché siano in grado di valutare e interagire criticamente con le informa- zioni provenienti dalla varietà dei media e delle fonti accademiche.

Se è vero, come asseriva Bourdieu (1986), che il capitale sociale è l'aggregato delle risorse effettive o potenziali che sono collegate al possesso di una rete duratura di relazioni più o meno istituzionalizzate di conoscenza reciproca, senza le abilità e le competenze necessarie per navigare in tali risorse, gli studenti si troverebbero in grande svantaggio. Il libero accesso alla grande quantità di informazioni di cui oggi disponiamo, cioè, non serve al progresso della società, se i membri che la compongono non sono in grado di distinguere le informazioni vere da quelle false, incomplete o obsolete. Ma anche, come prima accennato, di riconfigurare le proprie rappresentazioni sociali sulla realtà che generano comportamenti, scelte personali e stili di vita.

La formazione alla M.L. deve includere l'insegnamento agli studenti su come valutare e interpretare le informazioni, includendo le loro credenze e convinzioni su ciò che ritengono essere vero, ma riconoscendo anche pregiudizi che potrebbero sostenere le loro stesse credenze.

Diventare resilienti attraverso la M.L. significa, pertanto, imparare a rendersi consapevoli dei rischi di informazioni false, incomplete e obsolete cui siamo quotidianamente sottoposti e sapervi far fronte per essere in grado di esercitare il controllo sulle proprie scelte, decisioni e azioni, sia nell'ambito delle relazioni personali sia in quello della vita politica e sociale.

La responsabilizzazione dell'utente online è perseguibile solo a condizione che venga garantito un accesso libero e sostenibile alle informazioni e alle infrastrutture della società digitale. Viceversa, il rischio è quello di promuovere forme di controllo potenzialmente autoritarie.

L'ecosistema mediatico (Postman, 1970) deve essere indipendente e garantire il pluralismo, evitando, in questo senso, l'applicazione troppo rigida di normative (ad esempio, la direttiva europea sul diritto d'autore, che rischia da un lato di limitare il libero accesso alle notizie di qualità e dall'altro di favorire, paradossalmente, proprio la cattiva informazione). Infine, dobbiamo leggere la resilienza anche come una strategia fondamentale per la rimozione degli ostacoli che impediscono ai cittadini del web di partecipare appieno alla società digitale, evitando che la rete venga a configurarsi come un moltiplicatore delle disuguaglianze sociali e lavorando affinché tutti e ciascuno possano fornire il proprio contributo alla società della conoscenza. Quest'ultima, per essere in grado di trasformare la società dell'informazione in risorse che consentano di agire efficacemente per migliorare la condizione umana. 


\section{BIBLIOGRAFIA}

AGCOM (2018). Delibera n. 403/18/CONS. Avvio del procedimento per l'adozione di un regolamento in materia di rispetto della dignità umana e del principio di non discriminazione e di contrasto all'hate speech $e$ all'istigazione all'odio.

Article 19 (2015). 'Hate Speech' Explained. A Toolkit. Free Word Center.

Baacke, D. (1997). Medienpädagogik. Niemeyer.

Bourdieu, P. (1986). The Forms of Capital. In Richardson J. G. (Ed.), Handbook of theory and research for the sociology of education (pp. 241-258). Greenwood Press.

Buccolo, M., Ferro Allodola, V., \& Mongili, S. (in press). Percezioni e vissuti emozionali ai tempi del Covid-19: una ricerca esplorativa per riflettere sulle proprie esistenze. Lifelong Lifewide Learning.

Buckingham, D., Banaji, S., Carr, D., Cranmer, S., \& Willett, R. (2005). The media literacy of children and young people: a review of the research literature. Ofcom.

Cadalanu, G. (2020, Aprile 10). Razzismo e coronavirus: dall'Asia, all'Europa, agli Stati Uniti, la giostra di "scherzi linguistici" e battute ispirati dalla discriminazione. La Repubblica.

Cappello, G. (2019). Media Literacy in Italy. The International Encyclopedia of Media Literacy: 1-6. https:// doi.org/10.1002/9781118978238.ieml0155

Cappello, G., D’Abbicco, L. \& Ranieri, M. (2018). Editoriale. La media education nell'era della post-verità. Media Education - Studi, ricerche, buone pratiche, 9(1), II-V. https://doi.org/10.36253/me-8795

Censis (2017). 51 ${ }^{\circ}$ Rapporto sulla situazione sociale del Paese. FrancoAngeli.

Cosentino, G. (2017). L'era della post-verità. Media e populismo dalla Brexit a Trump. Imprimatur s.r.l.

EUROSTAT (2020). E-government activities of individuals via website (Last update: 15-04-2020).

Faloppa, F. (2020). Sul «nemico invisibile» e altre metafore di guerra. La cura delle parole, Enciclopedia Treccani.

Farr, R. M., \& Moscovici, S. (Eds.) (1989). Rappresentazioni sociali. Il Mulino.

Federanziani (2020). Vademecum di Senior Italia Federanziani. Come difendersi dal Coronavirus.

Felini, D. (2004). Pedagogia dei media. Questioni, percorsi e sviluppi. La Scuola.

Ferro Allodola, V. (2013). Rappresentazioni sociali e costruzione di identità professionali "riflessive" nei contesti psichiatrici: considerazioni formative. Studi Sulla Formazione/Open Journal of Education, 16(1), 121133. https://doi.org/10.13128/Studi_Formaz-13489
Ferro Allodola, V. (2020). Le fake news come "movimento sommerso" di protesta? Intervista a Ermes Maiolica. Pedagogika, 24(1), 56-62.

Gili, G., \& Maddalena, G. (2018). Post-verità e fake news: radici, significati attuali, inattesi protagonisti e probabili vittime. Media Education - Studi, ricerche, buone pratiche, 9(1), 1-16. https://doi.org/10.36253/ me-8796

Gori, U. (a cura di) (2018). Cyber Warfare 2017: Information, Cyber e Hybrid Warfare: contenuti, differenze, applicazioni. FrancoAngeli.

Grandi, N., \& Piovan, A. (2020, Marzo 26). I pericoli dell'infodemia. La comunicazione ai tempi del coronavirus, MicroMega on-line.

Hobbs, R. (2010). Digital and Media Literacy: A Plan of Action. The Aspen Institute.

House of Commons Digital, Culture, Media and Sport Committee (2019). Disinformation and 'fake news': Final Report Eighth Report of Session 2017-19.

International Press Institute - IPI (2019). Newsroom Best Practices for Addressing Online Violence against Journalists Perspectives from Finland, Germany, Poland, Spain and the United Kingdom. @globalfreemedia.

ISS (2020). Cosa sono $i$ coronavirus (on-line 23/02/2020).

Jansen, E., \& James, V. (2014). Netlingo: The Internet Dictionary. Netlingo Inc.

Lazer, D. M. J., Baum, M. A., Benkler, Y., Berinsky, A. J., Greenhill, K. M., Menczer, F., Metzger, M. J., Nyhan, B., Pennycook, G., Rothschild, D., Schudson, M., Sloman, S. A., Sunstein, C. R., Thorson, E. A., Watts, D. J., \& Zittrain J. L. (2018). The science of fake news. Addressing fake news requires a multidisciplinary effort. Science, 359(6380), 1094-1096. https://10.1126/science.aao2998

Lessenski, M. (2018). Common sense wanted resilience to 'post-truth' and its predictors in the new media literacy index 2018. Report of European Policies Initiative, Open Society Institute.

Livingstone, S. (2004). Media literacy and the challenge of new information and communication technologies. Communication Review, 7(1), 3-14. htt ps://10.1080/10714420490280152

Mackintosh, E., \& Kiernan, E. (2018). Finland is winning the war on fake news. What it's learned may be crucial to Western democracy. CNN, Special Report.

Neuvonen, M., Kivinen, K., \& Salo, M. (2018). Elections approach - are you ready? Fact-checking for educators and future voters. FactBar EDU.

Newman, N., Fletcher, R., Kalogeropoulos, A., Levy, D. A. L., \& Nielsen, R. K. (2018). Digital News Report 2018. Reuters Institute for the Study of Journalism. 
Nicodemo, F. (2017). Disinformazia. La comunicazione al tempo dei social media. Marsilio.

Pasta, S. (2018). Razzismi 2.0. Analisi socio-educativa dellodio online. Morcelliana.

Postman, N. (1970). The Reformed English Curriculum. In Eurich A. C. (Ed). High School 1980. The Shape of Future in American Secondary Education (pp. 160168). Pitman.

Quattrociocchi, W., \& Vicini, A. (2016). Misinformation. Guida alla società dell'informazione e della credulità. FrancoAngeli.

Ranieri, M., Di Stasio, M., \& Bruni, I. (2018). Insegnare e apprendere sulle fake news. Uno studio esplorativo in contesto universitario. Media Education - Studi, ricerche, buone pratiche, 9(1), 94-111. https://doi. org $/ 10.36253 / \mathrm{me}-8801$

Rehm, G. (2018). An Infrastructure for Empowering Internet Users to Handle Fake News and Other Online Media Phenomena. In Rehm G. \& Declerck T. (Eds.) Language Technologies for the Challenges of the Digital Age. GSCL 2017. Lecture Notes in Computer Science, vol 10713. Springer. https://doi.org/10.1007/978-3-319-73706-5

Sensi, G. (2017). Comunicare per costruire coesione sociale. Welfare Oggi, 5, 97-101.

Silverstone, R. (2007). Media and Morality: On the Rise of the Mediapolis. Polity Press.

Sorrentino, C. (2018). Il giornalismo ai tempi della postverità. European Journal Observatory.

Stanford History Education Group (2016). Executive Summery. Evaluating information: The Cornerstone of Civic Online Reasoning.

Swire, B., Ecker, U. K. H., \& Lewandowsky, S. (2017). The role of familiarity in correcting inaccurate information. Journal of Experimental Psychology: Learning, Memory, and Cognition, 43(12), 1948-1961. https:// doi.org/10.1037/xlm0000422

Trinchero, R. (2018). Contro la guerra cognitiva. Educare allo scetticismo attivo. Media Education - Studi, ricerche, buone pratiche, 9(1), 17-36. https://doi. org/10.36253/me-8797

Ireton C. \& Posetti J. (Eds) (2018). Journalism, 'Fake News' and Disinformation: A Handbook for Journalism Education and Training. UNESCO.

Veltri, G. (2018). La tempesta perfetta: social media, fake news e la razionalità limitata del cittadino. Media Education - Studi, ricerche, buone pratiche, 9(1), 37-56. https://doi.org/10.36253/me-8798

Vosoughi, S., Roy, D., \& Aral, S. (2018). The spread of true and false news online, Science, 359(6380), 11461151. https://10.1126/science.aap9559

Weedon, J. Nuland, W., \& Stamos, A. (2017). Information operations and Facebook. Facebook. 